Bei der Produktion in Freeport werden eine neue Synthese und eine fortschrittliche Verfahrenstechnologie zur Anwendung kommen. Endgültige Produktformulierungen werden sowohl in den Roche-Produktionszentren in den Vereinigten Staaten als auch in Europa erfolgen. Teile der Produktion des neuen $\mathrm{Be}$ triebes werden als Zwischenstufen zur Herstellung anderer Carotinoide genutzl werden. Die Produktionskapazität in Freeport wird sowohl den Inlandsbedarf als auch die Exportnachfrage abdecken.

Dass die 'Awareness' der Amerikaner gestiegen ist, zeigen auf eindrückliche Weise die von uns bei Gallup in Auftrag gegebenen 'Awareness'-Studien: Heute hat jeder zweite Amerikaner Kenntnis von Beta-Carotin und dessen Rolle in der Ernährung.
Damit wären eigentlich alle wichtigen 'P's' abgehandelt. Da bleibt noch ein kleines Element - der Preis - bei dem Roche eigentlich gerne vermeidet, dass man ungebührlich darüber spricht, indem die Preise so angesetzt werden, dass sie dem Kundennutzen entsprechen.

Zusammenfassend haben wir am Beispiel von Beta-Carotin gesehen

- wie Beta-Carotin-Produktformen entwickelt werden, um die Färbe-Bedürfnisse einzelner Segmente abzudecken (Bsp. 5\% Emulsion/Softdrink)

- wie Beta-Carotin in seiner Eigenschaft als Provitamin A eingesetzt wird (Pharma und Lebensmittel)
- wie Beta-Carotin im Tierernährungssektor eingesetzt wird (Fertilität, Wildverbiss)

- wie eine weltweite Marketingorganisation aufgebaut und unterhalten werden muss, um geographisch alle Märkte erschliessen zu können

- wie neue Forschungsergebnisse, die vorliegen oder demnächst vorliegen werden, umgesetzt werden, und wie das Wissen um den potentiellen Nutzen von BetaCarotin als eigenständiger Mikronährstoff frühzeitig verbreitet wird

- wie rechtzeitig die Investitionen an die Hand genommen werden müssen, um die anvisierten 'Produkt/Marktstrategien' auch erfolgreich durchführen zu können.

\title{
Von der Idee zum verkaufsfertigen Produkt
}

\author{
Manfred Rembold*
}

Benötigt diese Thematik überhaupt eine Darstellung im Rahmen einer Seminarveranstaltung? Ergibt sich die Ideenfindung und deren Umsetzung in ein Produkt nicht von selbst und automatisch? Nachfolgend soll versucht werden, ein Bild dieses «automatischen» Ganges zu geben, dargestellt am Beispiel der Entwicklung von Textilchemikalien.

Um eine Vorstellung über die Grösse der Forschungs- und Entwicklungsaufwendungen zu erhalten - die ja ausschliesslich im Hinblick auf Produkt- oder Technologieinnovation getätigt werden - sind in Fig. 1 die Zahlen genannt, die Ciba-Geigy in den Jahren 1987 und 1988 ausgewiesen (Fig. 1) hat. Sie zeigen deutlich, wie aufwendig es heute für multinationale, chemische Unternehmen geworden ist, sich die Chancen und Optionen für eine Beteiligung an der technischen Fortentwicklung zu erhalten. Die für den finanziellen Erfolg der Unternehmung verantwortlichen Manager verfolgen mit Aufmerksamkeit diese Entwicklung und Fragen nach der Effizienz und Innovationskraft der eigenen Forschungsabteilungen werden gestellt.

Im folgenden sollen einige wesentliche Gesichtspunkte erfolgreicher Produktentwicklungen aufgezeigt und an einem Entwicklungsbeispiel aus dem Gebiet der Textilchemikalien verdeutlicht werden. Zwei
Fragenkomplexe sind dabei besonders zu betrachten:

\section{Wie kommt es zur Ideenbildung? Woher sind Anregungen in der Zukunft zu er-} warten?

2. Worin liegen die Voraussetzungen für eine erfolgreiche Umsetzung der Idee? Produktewerdegang: Patentierung, Registrierung, Produktionseinführung.

Am Beispiel eines photochemischen Stabilisators für Polyamid sollen diese Fragen konkret beantwortet werden.

Für viele technische Güter, wie z.B. auch für Farbstoffe und Textilchemikalien, findet man heute Marktbedingungen vor, die - summarisch betrachtet - dadurch gekennzeichnet sind, dass die Abnehmerindustrien nur ein langsames oder gar kein Wachstum aufweisen, ja teilweise sogar einen Rückgang hinnehmen mussten. Eine Situation, die kennzeichnend für $\mathrm{Ge}$ schäfte mit hohem Reifegrad ist. Die Folgen sind hoher Kostendruck, gedämpfte Innovationsneigung - insbesondere wo hohe Investitionen notwendig würden und dies bedeutet, dass auch bei den Zulieferindustrien die Risikobereitschaft für Neuentwicklungen sinkt. Das heisst nicht, dass nicht in F \& E Projekte investiert würde, aber dies geschieht vor allem im

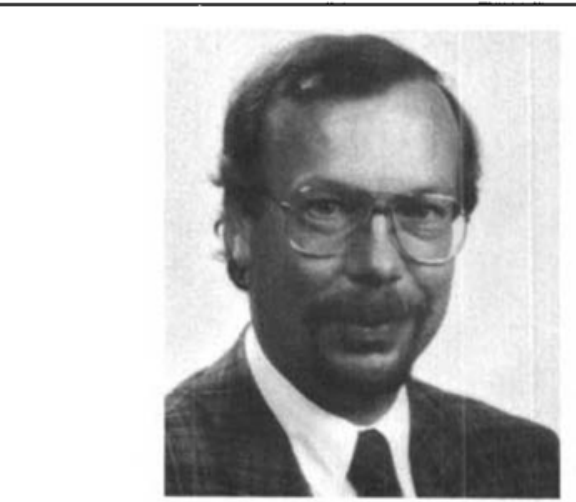

Manfred Rembold: Geboren 1950 in Stuttgart, BRD. Studium der Chemic an der Universität Stuttgart - Diplom 1975. Dissertation bei Prof. H.E.A. Kramer, Institut für Physikalische Chemie, über Singulettsauerstoffbildung durch Diaminoanthachinone und dessen Bedeutung für katalytische Ausbleichreaktionen von Farbstoffen. Nach der Promotion $1 \mathrm{Jahr}$ wissenschaftlicher Assistent am gleichen Institut. Seit 1980 bei Ciba-Geigy $A G$, Basel. Tätigkeiten: Anwendung und Produktentwicklung Stabilisatoren für Lacke; Anwendungsleiter Additive für die Strahlungshärtung. Seit 1989 Gruppenleiter Applikationsforschung: Chemikalien für die Textil- und Waschmittelindustrie.

Hinblick auf rasche Realisierbarkeit der Projekte, kaum in längerfristige exploratorische Bearbeitungen. Diese beinhalten zwar eine potentiell grosse Chance, aber eine zu geringe Wahrscheinlichkeit, dass die Chance auch gewinnbringend genutzt werden kann.

Worauf soll sich in einer solchen Situation Forschung konzentrieren, woher soll man die Anregungen nehmen und die richtigen, das heisst erfolgversprechenden Ideen verwirklichen? Betrachtet man die möglichen Einflüsse und Impulse für den in diesem Zweig der industriellen Forschung tätigen Chemiker (Fig. 2), so sind als erste Impulsgeber die technischen

\footnotetext{
* Korrespondenz: Dr. M. Rembold Ciba-Geigy $A G$

$\mathrm{CH}-4002$ Basel
} 
Bei der Produktion in Freeport werden eine neue Synthese und eine fortschrittliche Verfahrenstechnologie zur Anwendung kommen. Endgültige Produktformulierungen werden sowohl in den Roche-Produktionszentren in den Vereinigten Staaten als auch in Europa erfolgen. Teile der Produktion des neuen $\mathrm{Be}$ triebes werden als Zwischenstufen zur Herstellung anderer Carotinoide genutzl werden. Die Produktionskapazität in Freeport wird sowohl den Inlandsbedarf als auch die Exportnachfrage abdecken.

Dass die 'Awareness' der Amerikaner gestiegen ist, zeigen auf eindrückliche Weise die von uns bei Gallup in Auftrag gegebenen 'Awareness'-Studien: Heute hat jeder zweite Amerikaner Kenntnis von Beta-Carotin und dessen Rolle in der Ernährung.
Damit wären eigentlich alle wichtigen 'P's' abgehandelt. Da bleibt noch ein kleines Element - der Preis - bei dem Roche eigentlich gerne vermeidet, dass man ungebührlich darüber spricht, indem die Preise so angesetzt werden, dass sie dem Kundennutzen entsprechen.

Zusammenfassend haben wir am Beispiel von Beta-Carotin gesehen

- wie Beta-Carotin-Produktformen entwickelt werden, um die Färbe-Bedürfnisse einzelner Segmente abzudecken (Bsp. 5\% Emulsion/Softdrink)

- wie Beta-Carotin in seiner Eigenschaft als Provitamin A eingesetzt wird (Pharma und Lebensmittel)
- wie Beta-Carotin im Tierernährungssektor eingesetzt wird (Fertilität, Wildverbiss)

- wie eine weltweite Marketingorganisation aufgebaut und unterhalten werden muss, um geographisch alle Märkte erschliessen zu können

- wie neue Forschungsergebnisse, die vorliegen oder demnächst vorliegen werden, umgesetzt werden, und wie das Wissen um den potentiellen Nutzen von BetaCarotin als eigenständiger Mikronährstoff frühzeitig verbreitet wird

- wie rechtzeitig die Investitionen an die Hand genommen werden müssen, um die anvisierten 'Produkt/Marktstrategien' auch erfolgreich durchführen zu können.

\title{
Von der Idee zum verkaufsfertigen Produkt
}

\author{
Manfred Rembold*
}

Benötigt diese Thematik überhaupt eine Darstellung im Rahmen einer Seminarveranstaltung? Ergibt sich die Ideenfindung und deren Umsetzung in ein Produkt nicht von selbst und automatisch? Nachfolgend soll versucht werden, ein Bild dieses «automatischen» Ganges zu geben, dargestellt am Beispiel der Entwicklung von Textilchemikalien.

Um eine Vorstellung über die Grösse der Forschungs- und Entwicklungsaufwendungen zu erhalten - die ja ausschliesslich im Hinblick auf Produkt- oder Technologieinnovation getätigt werden - sind in Fig. 1 die Zahlen genannt, die Ciba-Geigy in den Jahren 1987 und 1988 ausgewiesen (Fig. 1) hat. Sie zeigen deutlich, wie aufwendig es heute für multinationale, chemische Unternehmen geworden ist, sich die Chancen und Optionen für eine Beteiligung an der technischen Fortentwicklung zu erhalten. Die für den finanziellen Erfolg der Unternehmung verantwortlichen Manager verfolgen mit Aufmerksamkeit diese Entwicklung und Fragen nach der Effizienz und Innovationskraft der eigenen Forschungsabteilungen werden gestellt.

Im folgenden sollen einige wesentliche Gesichtspunkte erfolgreicher Produktentwicklungen aufgezeigt und an einem Entwicklungsbeispiel aus dem Gebiet der Textilchemikalien verdeutlicht werden. Zwei
Fragenkomplexe sind dabei besonders zu betrachten:

\section{Wie kommt es zur Ideenbildung? Woher sind Anregungen in der Zukunft zu er-} warten?

2. Worin liegen die Voraussetzungen für eine erfolgreiche Umsetzung der Idee? Produktewerdegang: Patentierung, Registrierung, Produktionseinführung.

Am Beispiel eines photochemischen Stabilisators für Polyamid sollen diese Fragen konkret beantwortet werden.

Für viele technische Güter, wie z.B. auch für Farbstoffe und Textilchemikalien, findet man heute Marktbedingungen vor, die - summarisch betrachtet - dadurch gekennzeichnet sind, dass die Abnehmerindustrien nur ein langsames oder gar kein Wachstum aufweisen, ja teilweise sogar einen Rückgang hinnehmen mussten. Eine Situation, die kennzeichnend für $\mathrm{Ge}-$ schäfte mit hohem Reifegrad ist. Die Folgen sind hoher Kostendruck, gedämpfte Innovationsneigung - insbesondere wo hohe Investitionen notwendig würden und dies bedeutet, dass auch bei den Zulieferindustrien die Risikobereitschaft für Neuentwicklungen sinkt. Das heisst nicht, dass nicht in F \& E Projekte investiert würde, aber dies geschieht vor allem im

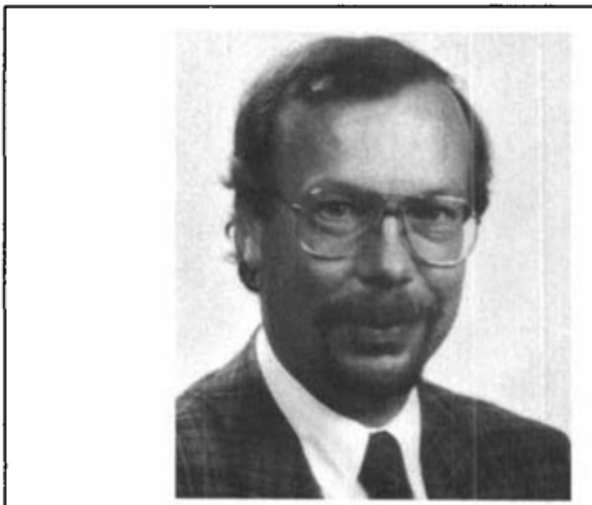

Manfred Rembold: Geboren 1950 in Stuttgart, BRD. Studium der Chemic an der Universität Stuttgart - Diplom 1975. Dissertation bei Prof. H.E.A. Kramer, Institut für Physikalische Chemie, über Singulettsauerstoffbildung durch Diaminoanthachinone und dessen Bedeutung für katalytische Ausbleichreaktionen von Farbstoffen. Nach der Promotion $1 \mathrm{Jahr}$ wissenschaftlicher Assistent am gleichen Institut. Seit 1980 bei Ciba-Geigy $A G$, Basel. Tätigkeiten: Anwendung und Produktentwicklung Stabilisatoren für Lacke; Anwendungsleiter Additive für die Strahlungshärtung. Seit 1989 Gruppenleiter Applikationsforschung: Chemikalien für die Textil- und Waschmittelindustrie.

Hinblick auf rasche Realisierbarkeit der Projekte, kaum in längerfristige exploratorische Bearbeitungen. Diese beinhalten zwar eine potentiell grosse Chance, aber eine zu geringe Wahrscheinlichkeit, dass die Chance auch gewinnbringend genutzt werden kann.

Worauf soll sich in einer solchen Situation Forschung konzentrieren, woher soll man die Anregungen nehmen und die richtigen, das heisst erfolgversprechenden Ideen verwirklichen? Betrachtet man die möglichen Einflüsse und Impulse für den in diesem Zweig der industriellen Forschung tätigen Chemiker (Fig. 2), so sind als erste Impulsgeber die technischen

\footnotetext{
* Korrespondenz: Dr. M. Rembold Ciba-Geigy $A G$

$\mathrm{CH}-4002$ Basel
} 


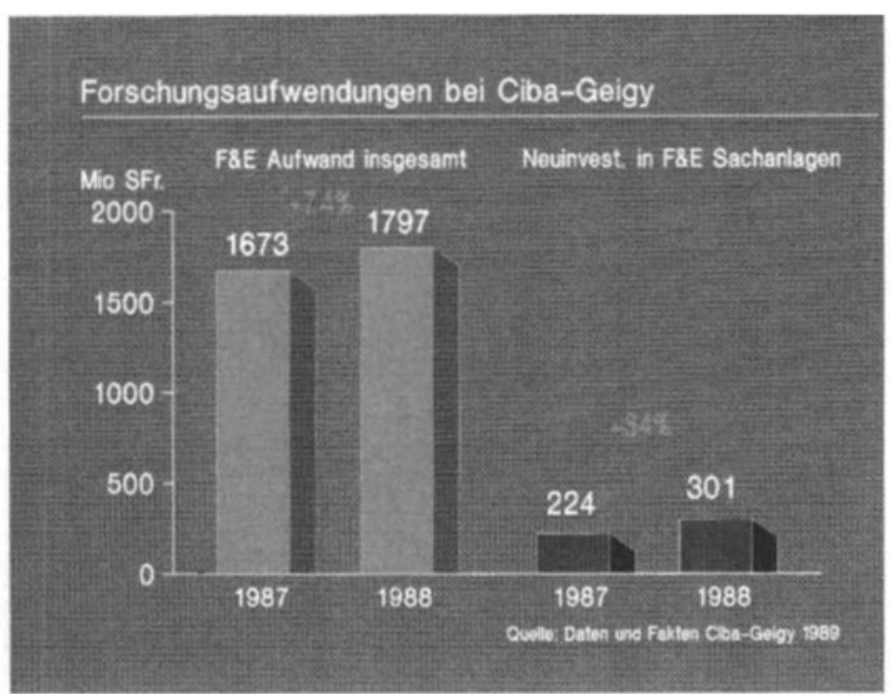

Fig. I

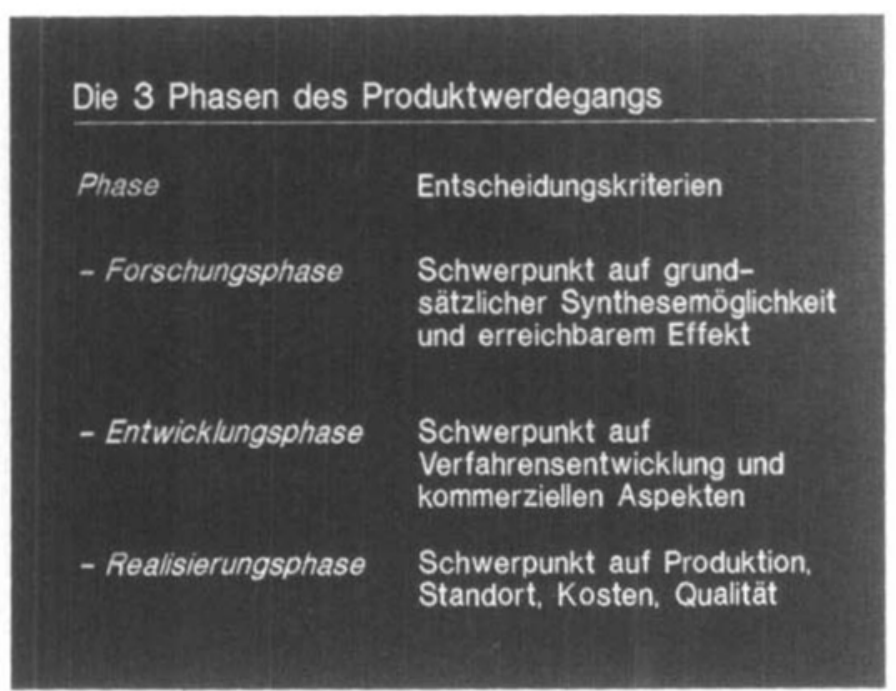

Fig. 3

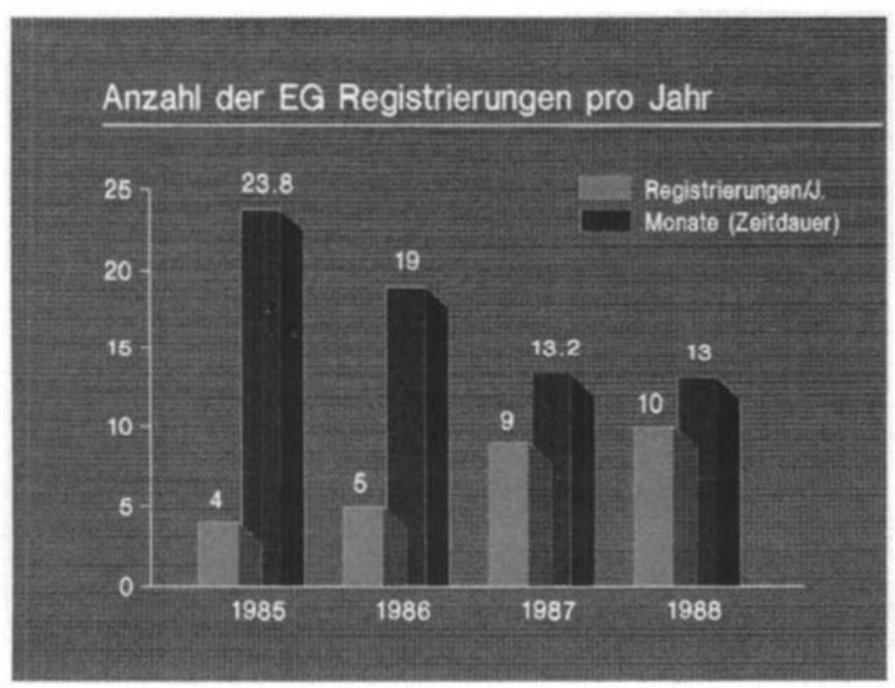

Fig. 5

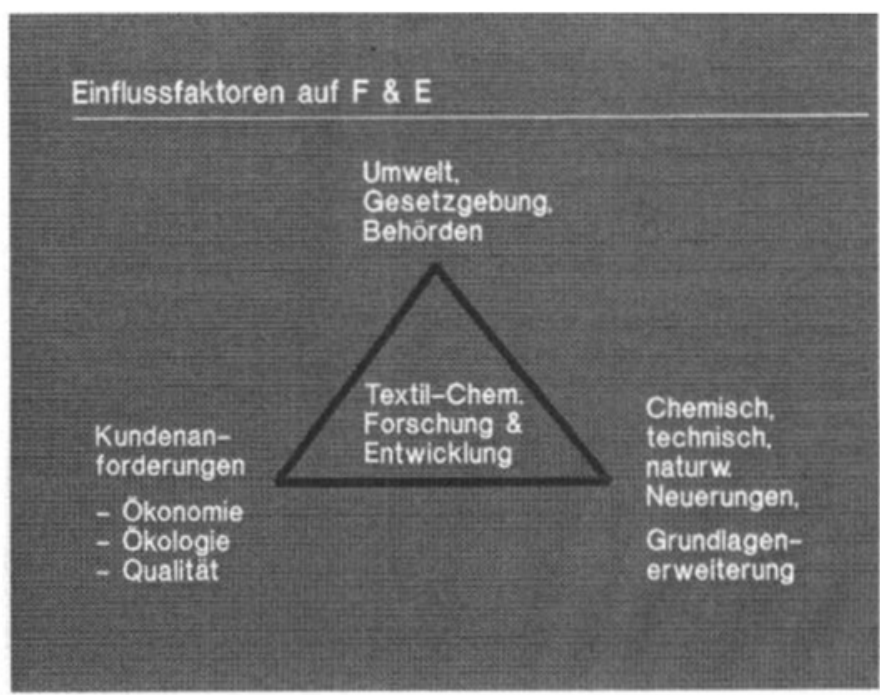

Fig. 2

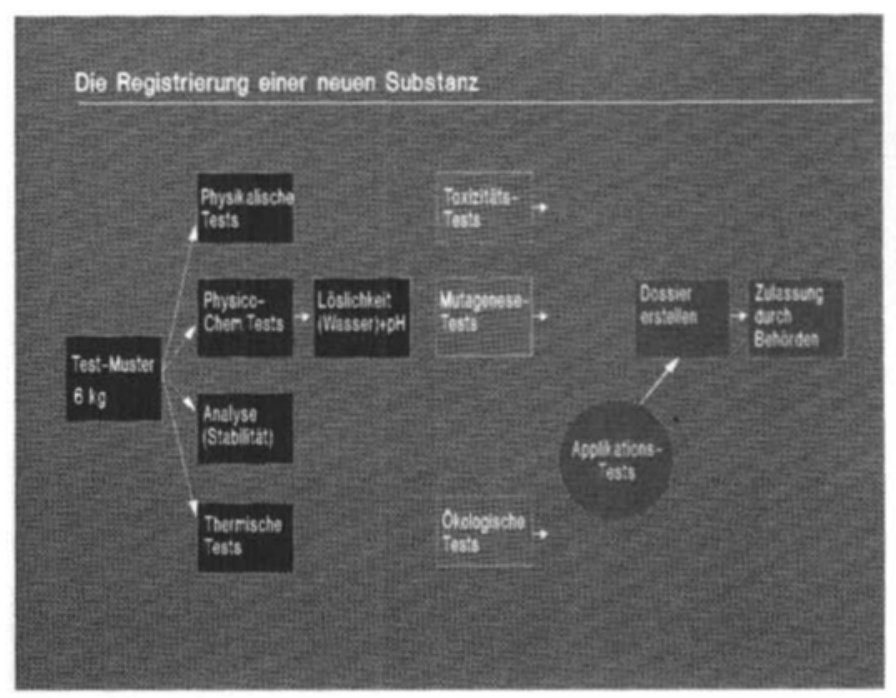

Fig. 4

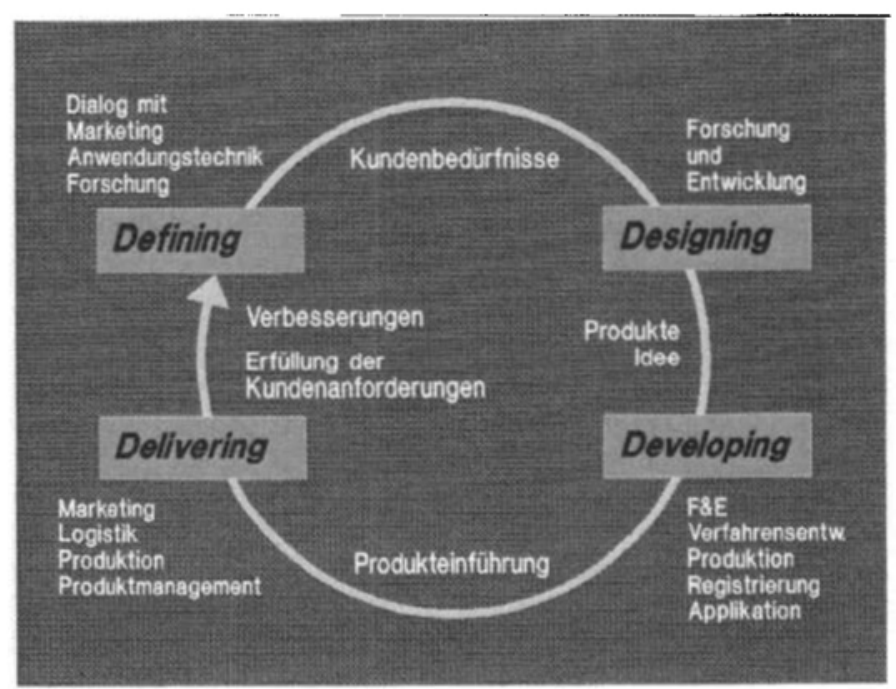

Fig. 6
Neuerungen und die Grundlagenerweiterung auf dem speziellen Gebiet der Textilchemikalien zu nennen. Grundsätzlich neue synthetische Fasern sind in der Bekleidungs- und Heimtextilienindustrie kaum zu erwarten, grundsätzlich neue chemische Strukturen auf dem Gebiet der Veredlungschemikalien für diese Segmente sind nicht zu sehen. Die Zusammenhänge sind im Groben erforscht, überraschend Neues, Abenteuerliches ist auf diesem Gebiet mit rein synthetisch chemischen Methoden wenig wahrscheinlich. Die Chance liegt eher im Nutzbarmachen von Technologien, die selbst noch stark im Fluss sind, und diese auf das Gebiet der Textilvered- lung zu übertragen. Beispiele hierfür könnten sein: Lasertechnologie zur Erzeugung neuer Effekte und Strukturen, UV-, Elektronen-Strahlung, Gasplasmen zur Fixierung von Ausrüstungen oder Modifizierung von Geweben, sowie die Nutzung überkritischer Systeme zur Applikation von Farbstoffen und Hilfsmitteln. 
Etwas anders verhält es sich mit textilen Materialien für technische Zwecke. Technische Textilien haben, je nach ihrem Verwendungszweck, sehr spezielle Aufgaben zu erfüllen und dies über einen langen Zeitraum. Hier kann die klassische Veredlungschemie einsetzen und in vielen Fällen den Gebrauch der Faser erst ermöglichen. Ein Beispiel dafür sind Chemikalien, die die UV-Lichtbeständigkeit von Synthesefasern und von Färbungen erhöhen.

Gesamthaft gesehen sollten also Innovationen eher von heute möglichen Technologien mit der Chemie als Partnerwissenschaft, als von rein synthetischen Arbeiten auf diesem Gebiet, kommen. Das soll aber nicht heissen, dass es in der synthetischen Chemie als Partnerwissenschaft nicht noch viel Interessantes zu tun gäbe. Auf dem Gebiet der Textilchemikalien bedeutet dies, das breite chemische Wissen zur interdisziplinären Lösung anwendungstechnischer Probleme einzusetzen, sowie die Herausarbeitung von Struktur-Wirkungsbeziehungen, um dadurch eventuell neue chemische Leitstrukturen zur Erreichung eines gewünschten Effektes zu finden.

Eine weitere wichtige Einflussgrösse ist der Anforderungskatalog, der von unseren Kunden gestellt wird. Drei Kundenanforderungen beeinflussen unsere Forschungsaktivitäten in besonderem Masse:

- Bestehende Produkte kostengünstiger zur Verfügung zu stellen, bzw. einfachere Herstellverfahren beim Kunden zu ermöglichen;

- qualitativ höherwertige Produkte oder Anwendungsverfahren zu ermöglichen, wobei eine sorgfältige Definition des Begriffs Qualität jeweils vorgenommen werden muss;

- ökologisch günstigere Produkte einzusetzen oder Verarbeitungsprozesse ökologisch zu verbessern.

Die Erfolge der chemischen Industrie auf dem Gebiet der Textilchemikalien, wissenschaftliche Erkenntnisse in relativ einfach zu handhabende Produkte umzusetzen und diese weltweit zu vermarkten, waren in der Vergangenheit gross. Andererseits sind durch die Anwendung einzelner Produkte in der Umwelt Probleme entstanden, deren Lösung und Beseitigung wiederum den Chemiker verlangt. Die Forderung nach mehr Schutz und Sicherheit für unsere Umwelt ist ein grosser Impulsgeber für unsere Forschung. In diese Richtung weist auch der Einfluss, den der Gesetzgeber mit behördlichen Auflagen ausüben kann. Auch von dieser Seite werden unter anderem ökologische Forderungen an uns gestellt, die wir als Chance für Neuentwicklungen auffassen müssen. So gesehen sind unsere Möglichkeiten zur Erforschung von Zusammenhängen und daraus zur gezielten Produktentwicklung heute gar nicht so schlecht, ja vielleicht ist die Klassifizierung der Textilchemikalien als reifes Geschäft bereits heute schon zum
Teil überholt oder trifft nur bei stark vereinfachter Betrachtung zu.

Wie lässt sich nun in einem grossen Unternehmen gewährleisten, dass möglichst alle diese Anregungen aufgenommen, möglichst optimal entwickelt und zu Produkten umgesetzt werden? Was den wichtigen Teil der Kundenwünsche betrifft, kommen dem Mitarbeiter im Aussendienst und dem Mitarbeiter im Marketing eine wichtige Rolle $\mathrm{zu}$. Er ist das Bindeglied zum Kunden und trägt einen wesentlichen Teil der Verantwortung dafür, dass dessen Bedürfnisse richtig in den Forschungsbereich gelangen und dort in eine Zielsetzung münden. Das soll nicht heissen, dass kein direkter Kontakt zwischen Kunden und Forschung und Entwicklung bestehen soll. Im Gegenteil, dies ist in manchen Fällen äusserst notwendig; vor allem, wenn es um die Frage der Machbarkeit neuer Ideen geht, um die Abschätzung, ob mit der im Hause betriebenen Chemie eine Problemlösung für den Kunden erreicht werden kann.

Der ganze Komplex stellt ein schwieriges Kommunikationsproblem dar, das nur durch gute Zusammenarbeit von Marketing, Applikation, Forschung und Entwicklung gemeinsam gelöst werden kann. Persönliche Beziehungen, gute oder schlechte, können das Ergebnis sehr wesentlich beeinflussen. Ein Aspekt, der bei der Besetzung eines Projektteams sehr hoch eingeschätzt werden muss. Erfolgreiches Erforschen und Entwickeln von Produkten findet heute auf diesem Gebiet in den seltensten Fällen durch Einzellösungen statt, sondern ist das Produkt gemeinsamer fachübergreifender Anstrengungen. Die Festlegung des Zieles gehört dabei zu den schwierigsten Aufgaben.

Mit diesem Bekenntnis zur Teamarbeit soll jedoch nicht die andere Seite der Medaille unterschlagen werden, nämlich die Tatsache, dass viele Anstösse für neue Entwicklungen ihren Anfang in Einzelaktionen von kreativen Individualisten in $F$ \& $\mathrm{E}$ und Marketing nehmen. Ohne Zweifel stehen jedoch Teamfähigkeit und Teamgeist im Vordergrund, wenn es um die Entwicklung und Realisierung der einmal gefundenen Produktidee geht.

Welchen Weg muss eine Produktidee bis zu ihrer Realisierung zurücklegen? Dies ist im Produktewerdegang festgelegt. Darin werden die Aufgaben der Bereiche Forschung \& Entwicklung, Marketing und Produktion festgelegt. Es wird klar definiert, wer zu welchem Zeitpunkt Informationen und Arbeitsergebnisse bereitstellen muss.

Ein wesentliches Merkmal in unserem Fall ist die Gliederung in drei Phasen, nach denen jeweils über die Weiterbearbeitung entschieden werden muss. Diese drei Phasen bestehen aus einer Forschungsphase, einer Entwicklungsphase und einer Realisierungsphase (Fig. 3).

Während der Forschungsphase sollen eine chemische Leitstruktur gefunden oder eine bereits erkannte Leitstruktur gezielt derivatisiert werden, um das gewünschte applikatorische Anforderungsprofil zu erreichen. Die grobe Abklärung der Zugänglichkeit von Ausgangsverbindungen und die Machbarkeit der Synthese sollten ebenfalls in dieser ersten Phase abgeklärt werden.

Die Entwicklungsphase, die sich daran anschliesst, beinhaltet nun schon die Ausarbeitung und Optimierung des Herstellungsverfahrens und die Aufgabe der Registrierung.

Die dritte Phase nennen wir Realisierungsphase, hier geht es hauptsächlich um Produktionsfragen, wie Standort, Produktekosten, Bedarf und endgültige Aufnahme ins Sortiment.

Diese Grobrasterung soll für die einzelnen Aspekte noch etwas vertieft werden. Ein äusserst wichtiger Punkt ist die Erstellung der Patentlage, bzw. die Neuanmeldung entweder der neuen Verbindungen (d.h. Stoffschutz) oder des an sich bekannten Stoffes in einer neuen Verwendung oder, als dritte Möglichkeit, spezielle Schritte des Herstellungsverfahrens. Im allgemeinen wird man, wenn immer möglich, den Stoffschutz anstreben, da dies für ein Handelsprodukt die besten Schutzrechte bietet. In vielen Fällen ist dies jedoch nicht mehr möglich, da die Verbindungen an sich bekannt sind und nur für den vorgesehenen Verwendungszweck neu eingesetzt werden sollen. In diesem Fall lässt sich mit einem Applikationspatent, in dem die Verwendung geschützt ist, häufig noch ein ausreichend guter Schutz vor Nachahmung bzw. Umgehung erzielen. Kann überhaupt kein Schutzrecht für die beabsichtigte Applikation mehr in Anspruch genommen werden, so ist sehr sorgfältig zu prüfen, ob eine Weiterbearbeitung dieses Projektes überhaupt noch sinnvoll ist.

Neben der Patentierung müssen erste toxikologische Prüfungen, erste Verfahren zur Herstellung in grösserem Maßstab, erste ökologische Beurteilungen, die $\mathrm{Zu}$ gänglichkeit der Ausgangsverbindungen und schliesslich der Registrierstatus abgeklärt werden. Für die Registrierung ist es von grosser Bedeutung, ob es sich um bekannte, bereits zugelassene Stoffe oder um neue Stoffe handelt.

Man sieht, dass bereits in diesem frühen Stadium des Produktewerdeganges die Dienste vieler Abteilungen in Anspruch genommen werden müssen, was die oben erwähnte Teamfähigkeit und Kooperationsbereitschaft aller Beteiligten voraussetzt.

Ein ebenfalls äusserst wichtiger Aspekt, der insbesondere während der Entwicklungsphase in den letzten Jahren noch an Bedeutung gewonnen hat, ist der ökologische Aspekt.

Er kommt in zweifacher Form im Produktewerdegang zum Ausdruck:

1. Es muss eine Gesamtbeurteilung der ökologischen Situation des gewählten Herstellungsverfahrens sowie für die vorgesehene Anwendung erstellt wer- 
den. Darunter fallen insbesondere $\mathrm{Ab}$ wasserlasten bei der Produktion und der Anwendung des Produktes beim Kunden, sowie mögliche Immissionen über die Abluft und ebenso die Deponierbarkeit der festen Abfälle.

2. Bei der Registrierung im Bereich Produktesicherheit, wo die relevanten ökologischen und toxikologischen Daten erstellt werden.

Nach der Registrierung und Zulassung durch die Behörden tritt das Produkt ans Licht der Öffentlichkeit. Ziel der Registrierung ist eine Beurteilung des Produktes hinsichtlich seiner ökologischen, toxikologischen und für die Arbeitssicherheit relevanten Eigenschaften. Diese Unterlagen werden den Behörden zur Genehmigung vorgelegt, sie bilden die Entscheidungsgrundlage.

Die durchzuführenden Prüfungen lassen sich in zwei Blöcke gliedern (Fig. 4).

1. Prüfungen, die sich mit den physikalischen und physikalisch chemischen Eigenschaften des Produktes befassen.

2. Prüfungen, die sich mit dem befassen, was geschieht, wenn das Produkt mit Mensch, Tier oder mit Wasser, Luft, Boden in Berührung kommt.

Um alle diese Prüfungen durchführen zu können, werden bereits Kilogramm-Mengen benötigt. Dieses Muster muss repräsentativen Charakter für die spätere Handelsware haben, was für Reaktionsmischungen, die von der Prozessführung abhängen, recht problematisch sein kann.

Wenn man die Gesamtheit der durchzuführenden Prüfungen, insbesondere der toxikologischen Prüfungen, betrachtet, ist zweierlei sehr auffällig:

1. Es muss ein hoher Aufwand im ökologischen und toxikologischen Bereich betrieben werden, um eine möglichst hohe Produktesicherheit zu gewährleisten, und

2. es sind eine Vielzahl von Kenntnissen und Fähigkeiten notwendig, um die verschiedenen Prüfungen überhaupt durchführen zu können. Hinter beinahe jeder Testmethode steht ein kleines Team hochspezialisierter Chemiker und Biologen, die einen Teilbeitrag an der Produktentwicklung leisten.

Das Resultat aller Prüfungen ist dann ein Dossier, anhand dessen die Behörden eine Entscheidung über die Zulassung des Produktes fällen können. Dieses Dossier muss für Japan, USA sowie Europa separat eingereicht werden, wobei die Daten nur einmal erstellt werden müssen, formale Änderungen und Ergänzungen aber durchaus auftreten können.
Vielfach findet man die Ansicht vertreten, dass die Registrierung zum geschwindigkeitsbestimmenden Schritt bei der Markteinführung geworden ist. Dies trifft für unseren Bereich nicht zu (Fig. 5) Während 19854 Registrierungen knapp 2 Jahre dauerten, hat sich dies 3 Jahre später deutlich gewandelt. 1988 durchliefen 10 Produkte in 13 Monaten den Prozess.

Das konkrete Beispiel einer neuen Produktidee, die heute als Veredlungschemikalie für Polyamidtextilien weltweit auf dem Markt ist und hauptsächlich in der Automobilausstattung verwendet wird, soll die obigen Entwicklungsschritte noch einmal verdeutlichen. Seit vielen Jahren weiss man, dass Polyamidfasern und daraus hergestellte Gewebe bei Lichteinwirkung - insbesondere UV-Strahlung, verbunden mit Wärme - rasch altern und ihre Gebrauchseigenschaften verlieren. Dies äussert sich in einem Verlust der ästhetischen Qualitäten wie Farbton, Oberfläche, aber - noch weit schlimmer - in den mechanischen Eigenschaften. Zugfestigkeit, Scheuerfestigkeit u.a. gehen mit der Zeit völlig verloren, das Gewebe zerfällt nach genügend langer Belichtungszeit praktisch schon beim Anfassen. Dies begrenzt die Lebensdauer solcherart hergestellter Artikel beträchtlich und hat in der Vergangenheit hauptsächlich bei Automobilausstattungen zu Reklamationen geführt.

Für die meisten Konsum- und Modeartikel ist dies in aller Regel ohne Bedeutung, da sie ohnehin nur kurze Zeit in Gebrauch sind. Die Situation ändert sich im Falle technischer Textilien. Dazu gehören Seile, Sicherheitsgurte, Autopolster, Autoteppiche und anderes mehr. In diesen Fällen ist die mechanische Beanspruchung der Textilien gross, und es kommt wesentlich darauf an, dass die mechanischen Qualitäten erhalten bleiben. Daher hat man schon seit einigen Jahren versucht, diese Artikel bei der Färbung durch den Zusatz von Stabilisatoren so weit in ihren Eigenschaften zu verbessern, dass sie den gestellten Anforderungen genügen. Eine Klasse von Verbindungen waren anorganische $\mathrm{Cu}$ Salze. Damit erreicht man zwar den gewünschten Effekt, handelt sich aber grosse ökologische Probleme ein, da anorganische Salze in hohen Konzentrationen eingesetzt werden müssen und diese einen sehr schlechten Aufziehgrad auf die Faser haben; d.h. die überwiegende Menge des Cu-Salzes bleibt in der wässrigen Flotte und muss, da die Einleitung von $\mathrm{Cu}$-Salzen in offene Gewässer strengen Limiten unterliegt, bei der Abwasserreinigung mühsam und teuer wieder entfernt werden.

Dadurch war ein ökologischer und ökonomischer Anreiz für eine Produktinnovation gegeben. Für uns war daher die Fragestellung neu so zu formulieren: Können wir eine Textilchemikalie herstellen und entwickeln, die verfahrenstechnisch breit einsetzbar ist, die deutlich besser auf die Polyamidfaser aufzieht und im ppm-Bereich wirksam ist? Dieses Grobanforderungsprofil wurde zwischen $F$ \& $E$ und Marketing 1984 als gemeinsames Ziel formuliert.

Die Verknüpfung bereits vorhandener Kenntnisse aus der Spinnmassenstabilisierung von Polyamid, verbunden mit den Kenntnissen der färberischen Anforderungen, der Applikation aus wässriger Flotte, führte Anfang 1985 zur Patentierung einer speziellen Klasse von $\mathrm{Cu}$-Komplexen für dieses Einsatzgebiet, die dadurch charakterisiert sind, dass sie einen hohen Aufziehgrad auf die Faser aufweisen und eine extrem niedrige Einsatzkonzentration erlauben.

Kurz darauf wurde mit der Registrierung begonnen, die sich damals noch bis Anfang 1987 erstreckte. Seit Ende 1986 wurde mit der Kundenbemusterung begonnen; im Laufe von 1988 begannen die Verkäufe hauptsächlich nach USA, und Anfang 1989 wurde das Produkt, Cibafast $\mathrm{N}$, in das Sortiment aufgenommen. Das heisst, der gesamte Weg wurde in etwa 3 Jahren durchlaufen, was unserer Meinung nach - gemessen an den zu überwindenden Schwierigkeiten - eine kurze Spanne ist.

Dieser Weg einer Produktidee zum verkaufsfertigen Produkt lässt sich in einer anderen Darstellung anschaulich zusammenfassen (Fig. 6). A.B. Cohan hat dies die 4 D's des Produktezyklus genannt: Defining, Designing, Developing, Delivering. Am Anfang stehen die Bedürfnisse des Kunden bzw. seine Anforderungen. Diese müssen im Dialog mit Marketing, Anwendungstechnik und $F$ \& $E$ möglichst exakt formuliert werden. Hier liegt der Schlüssel zum Erfolg, denn es nützt nichts, Produktziele zu formulieren und daran zu arbeiten, wenn diese nicht den echten Bedürfnissen des Kunden entsprechen.

Im nächsten Schritt, dem Designing, liegt die klassische Aufgabe der Forschung, Ideen zur Problemlösung zu entwickeln, Einsatzkonzepte aufzustellen und zu überprüfen.

Developing stellt den vielleicht interdisziplinärsten Schritt dar, da hier viele Bereiche involviert sind wie Entwicklung, Produktion, Analytik, Formulierung und Marketing.

Das Schlussziel ist dann die Lieferbereitschaft mit konstant hoher Qualität. Logistik, Produktion, Produktmanagement und Marketing übernehmen hier die Verantwortung.

Damit wäre der Kreis geschlossen, und es zeigt sich nun, ob im ersten Schritt wirklich die richtigen Kundenbedürfnisse formuliert worden sind und das neue Produkt am Markt erfolgreich sein wird. 